\title{
Strong uniqueness for stochastic evolution equations with unbounded measurable drift term
}

\author{
G. Da Prato* \\ Scuola Normale Superiore \\ Piazza dei Cavalieri 7, 56126 Pisa, Italy \\ F. Flandoli ${ }^{\dagger}$ \\ Dipartimento di Matematica Applicata "U. Dini" \\ Università di Pisa, Italy \\ E. Priola $\ddagger$ \\ Dipartimento di Matematica "G. Peano", Università di Torino \\ via Carlo Alberto 10, Torino, Italy \\ M. Röckner § \\ Faculty of Mathematics, Bielefeld University \\ 33501 Bielefeld, Germany
}

March 4, 2022

\begin{abstract}
We consider stochastic evolution equations in Hilbert spaces with merely measurable and locally bounded drift term $B$ and cylindrical Wiener noise. We prove pathwise (hence strong) uniqueness in the class of global solutions. This paper extends our previous paper [5] which generalized Veretennikov's fundamental result to infinite dimensions assuming boundedness of the drift term. As in [5] pathwise uniqueness holds for a large class, but not for every initial condition. We also include an application of our result to prove existence of strong solutions when the drift $B$ is only measurable, locally bounded and grows more than linearly.
\end{abstract}

Key words: Pathwise uniqueness, stochastic PDEs, locally bounded measurable drift term, strong mild solutions

Mathematics Subject Classification 35R60, 60H15

\footnotetext{
*E-mail: g.daprato@sns.it

${ }^{\dagger}$ E-mail: flandoli@dma.unipi.it

${ }_{\ddagger}^{\ddagger}$ E-mail: enrico.priola@unito.it

${ }^{\S}$ Research supported by the DFG through IRTG 1132 and CRC 701 and the I. Newton Institute, Cambridge, UK. E-mail: roeckner@mathematik.uni-bielefeld.de
} 


\section{Introduction}

We consider the following abstract stochastic differential equation in a separable Hilbert space $H$

$$
d X_{t}=\left(A X_{t}+B\left(X_{t}\right)\right) d t+d W_{t}, \quad t \geq 0, \quad X_{0}=x \in H,
$$

where $A: D(A) \subset H \rightarrow H$ is self-adjoint, negative definite and such that $(-A)^{-1+\delta}$, for some $\delta \in(0,1)$, is of trace class, $B: H \rightarrow H$ and $W=\left(W_{t}\right)$ is a cylindrical Wiener process. In [5] under the assumption that $B$ is Borel measurable and (globally) bounded we prove pathwise uniqueness of solutions to (1). A natural generalization is to extend it to the case where we only assume that $B$ is Borel measurable and locally bounded (i.e., bounded on balls):

$$
B \in B_{b, l o c}(H, H) .
$$

In this paper we prove that assuming (2) pathwise uniqueness holds for $\mu$-a.e. initial condition $x$ in the class of global mild solutions to (1). Here $\mu$ denotes the Gaussian measure which is invariant for the Ornstein-Uhlenbeck process $Z=\left(Z_{t}\right)$ which solves (11) when $B=0$ (see Section 1.1 for more details).

In other words if for some initial condition $x \in H, \mu$-a.e., there exists a solution for (1) on some filtered probability space $\left(\Omega, \mathcal{F},\left(\mathcal{F}_{t}\right), \mathbb{P}\right)$ with a cylindrical $\left(\mathcal{F}_{t}\right)$-Wiener process $W$ then our main result shows that this solution is pathwise unique. This is in particular the case when

$$
B \text { is measurable and at most of linear growth }
$$

(i.e., $B$ is measurable and there exists $a, b \geq 0$ such that $|B(x)| \leq a+b|x|, x \in H)$, because then existence of weak mild solutions is well-known (see Chapter 10 in [6], [11, 14] and also Appendix in [5]). Moreover, under condition (3), the unique law of any mild solution $X^{x}$ is equivalent to the law of the Ornstein-Uhlenbeck process starting at $x$ (corresponding to $B=0$ ).

By our main result, using a generalization of the Yamada-Watanabe theorem (see [18, 21]), one deduces that under (3) equation (11) has a unique strong mild solution, for $\mu$-a.e. $x \in H$, generalizing A. Veretennikov's seminal result [26] in the case $H=\mathbb{R}^{d}$ (see also [9, 10, 15, 16, 17, 25, 27, 28]) to infinite dimensions.

In Section 4 we generalize this by relaxing assumption (3). We prove existence of strong mild solutions, starting from $\mu$-a.e. initial condition $x \in H$, when $B \in$ $B_{b, l o c}(H, H)$ and moreover there exist $C>0, p>0$, such that

$$
\langle B(y+z), y\rangle \leq C\left(|y|^{2}+e^{p|z|}+1\right)
$$

for all $y, z \in H$ (see also Remark [17). Finally in Section 4.3 we show a possible extension of our result by considering local mild solutions.

In order to prove pathwise uniqueness for (10) we will consider bounded truncated drifts like

$$
B_{N}=B 1_{B(0, N)}, \quad N \geq 1
$$


where $B(0, N)$ is the open ball of center 0 and of radius $N$ and $1_{B(0, N)}$ is the indicator function of $B(0, N)$, by performing a suitable stopping time argument. This argument is not straightforward since it must be also used in combination with the Ito-Tanaka trick from [5] (see also [8, 12, 13, 23, 26]).

In addition in this paper we also simplify some arguments used in [5] in the case of $B \in B_{b}(H, H)$ (see, in particular, Lemma 8). Before stating our main result precisely, let us recall the following definition (cf. [21] and [18]).

Definition 1. Let $x \in H$.

(a) We call weak mild solution to (1) a tuple $\left(\Omega, \mathcal{F},\left(\mathcal{F}_{t}\right), \mathbb{P}, W, X\right)$, where $\left(\Omega, \mathcal{F},\left(\mathcal{F}_{t}\right), \mathbb{P}\right)$ is a filtered probability space on which it is defined a cylindrical $\left(\mathcal{F}_{t}\right)$-Wiener process $W$ and a continuous $\left(\mathcal{F}_{t}\right)$-adapted $H$-valued process $X=\left(X_{t}\right)=\left(X_{t}\right)_{t \geq 0}$ such that, $\mathbb{P}$-a.s.,

$$
X_{t}=e^{t A} x+\int_{0}^{t} e^{(t-s) A} B\left(X_{s}\right) d s+\int_{0}^{t} e^{(t-s) A} d W_{s}, \quad t \geq 0 .
$$

(b) A weak mild solution $X$ which is $\left(\overline{\mathcal{F}}_{t}^{W}\right)$-adapted (here $\left(\overline{\mathcal{F}}_{t}^{W}\right)$ denotes the completed natural filtration of the cylindrical process $W$ ) is called strong mild solution.

We will often use stopping times

$$
\tau_{N}^{X}=\inf \left\{t \geq 0: X_{t} \notin B(0, N)\right\}
$$

$\left(\tau_{N}^{X}=+\infty\right.$ if the set is empty), $N \geq 1$.

In our main result we consider two mild solutions $X$ and $Y$, having the same initial condition $x \in H$, and solving the same equation (11) but with possibly different drift terms, respectively $B$ and $B^{\prime} \in B_{b, l o c}(H, H)$, i.e.,

$$
\begin{gathered}
d X_{t}=\left(A X_{t}+B\left(X_{t}\right)\right) d t+d W_{t}, \quad X_{0}=x \\
d Y_{t}=\left(A Y_{t}+B^{\prime}\left(Y_{t}\right)\right) d t+d W_{t}, \quad Y_{0}=x
\end{gathered}
$$

Theorem 1. Assume Hypothesis 1 (see Section 1.1) and let $\mu$ be the centered Gaussian measure on $H$ with covariance $Q=-\frac{1}{2} A^{-1}$.

Then for $\mu$-a.e. $x \in H$, if $X$ and $Y$ are two weak mild solutions, respectively of (8) and (9), defined on the same filtered probability space $\left(\Omega, \mathcal{F},\left(\mathcal{F}_{t}\right), \mathbb{P}\right)$ with the same cylindrical Wiener process $W$, and if, for some $N \geq 1$,

$$
B(x)=B^{\prime}(x), \quad x \in B(0, N),
$$

then, $\mathbb{P}$-a.s.,

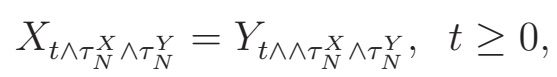

and so $\tau_{N}^{X}=\tau_{N}^{Y}, \mathbb{P}$-a.s.. 
Above we restrict to $W$ which are cylindrical with respect to the eigenbasis of $A$ (see Section 1.1 for details). Clearly if $B=B^{\prime}$ the result implies that, $\mathbb{P}$-a.s.,

$$
X_{t}=Y_{t}, \quad t \geq 0 .
$$

Indeed using that $\tau_{N}^{X} \uparrow+\infty$ and $\tau_{N}^{Y} \uparrow+\infty$ as $N \rightarrow \infty$ (because $X$ and $Y$ are both global solutions) we deduce easily (12) from (11).

The proof of Theorem 1, performed in Section 3, uses a truncation argument and regularity results for elliptic equations in Hilbert spaces involving truncated drift terms $B_{N}$ (cf. (5)). Such regularity results are given in Section 2, where we also establish an Itô type formula involving $u\left(X_{t}\right)$ with $u$ in some Sobolev space associated to $\mu$ (see Theorem [10). In comparison with [5] to prove such an Itô type formula we use a new analytic lemma (see Lemma 8).

There are several other quite essential differences in comparison with [5] in our proof. We refer to Remarks 9 and 12 for details.

\subsection{Assumptions and preliminaries}

As in [5] we consider a real separable Hilbert space $H$ and denote its norm and inner product by $|\cdot|$ and $\langle\cdot, \cdot\rangle$ respectively. Following [4], 6], [7], [22], we assume

Hypothesis $1 A: D(A) \subset H \rightarrow H$ is a negative definite self-adjoint operator and $(-A)^{-1+\delta}$, for some $\delta \in(0,1)$, is of trace class.

Remark 2. Our uniqueness result continues to hold under the following more general assumption: $A: D(A) \subset H \rightarrow H$ is self-adjoint and there exists $\omega \in \mathbb{R}$ such that $(A-\omega)$ is negative definite and $(\omega-A)^{-1+\delta}$, for some $\delta \in(0,1)$, is of trace class. Indeed if we write equation (11) in the form

$$
d X_{t}=\left(A X_{t}-\omega X_{t}\right) d t+\left(\omega X_{t}+B\left(X_{t}\right)\right) d t+d W_{t}, \quad X_{0}=x \in H,
$$

then the linear operator $(A-\omega I)$ verifies Hypothesis 1 and the drift $\omega I+B$ continues to satisfy (2).

Since $A^{-1}$ is compact, there exists an orthonormal basis $\left(e_{k}\right)$ in $H$ and a sequence of positive numbers $\left(\lambda_{k}\right)$ such that

$$
A e_{k}=-\lambda_{k} e_{k}, \quad k \in \mathbb{N} .
$$

Recall that $A$ generates an analytic semigroup $e^{t A}$ on $H$ such that $e^{t A} e_{k}=e^{-\lambda_{k} t} e_{k}$.

From now on until and including Section 3 we fix $\left(\Omega, \mathcal{F},\left(\mathcal{F}_{t}\right), \mathbb{P}\right), W, X$ and $Y$ as in the assertion of Theorem 1. As said before we will consider a cylindrical Wiener process $\left(W_{t}\right)$ with respect to the previous basis $\left(e_{k}\right)$. The process $\left(W_{t}\right)$ is formally given by " $W_{t}=\sum_{k \geq 1} \beta_{k}(t) e_{k}$ " where $\left(\beta_{k}(t)\right)$ are independent one-dimensional Wiener processes (see [6] for more details). 
By $R_{t}$ we denote the Ornstein-Uhlenbeck semigroup in $B_{b}(H)$ (the Banach space of all Borel and bounded real functions endowed with the essential supremum norm $\left.\|\cdot\|_{0}\right)$ defined as

$$
R_{t} f(x)=\int_{H} f(y) N\left(e^{t A} x, Q_{t}\right)(d y), \quad f \in B_{b}(H), x \in H,
$$

where $N\left(e^{t A} x, Q_{t}\right)$ denotes the Gaussian measure in $H$ of mean $e^{t A} x$ and covariance operator $Q_{t}$ given by

$$
Q_{t}=-\frac{1}{2} A^{-1}\left(I-e^{2 t A}\right), \quad t \geq 0 .
$$

We note that $R_{t}$ has the unique invariant measure $\mu:=N(0, Q)$ where $Q=-\frac{1}{2} A^{-1}$. Moreover, since under the previous hypotheses, the Ornstein-Uhlenbeck semigroup is strong Feller and irreducible we have by Doob's theorem that, for any $t>0, x \in H$, the measures $N\left(e^{t A} x, Q_{t}\right)$ and $\mu$ are equivalent; see [7]. On the other hand, our assumption that $(-A)^{-1+\delta}$ is trace class guarantees that the OU process

$$
Z_{t}=Z(t, x)=e^{t A} x+\int_{0}^{t} e^{(t-s) A} d W_{s}
$$

admits a continuous $H$-valued version.

If $H$ and $M$ are real separable Hilbert spaces, the Banach space $L^{p}(H, \mu ; M), p \geq 1$, is defined to consist of equivalent classes of measurable functions $\varphi: H \rightarrow M$ such that $\int_{H}|\varphi(x)|_{M}^{p} \mu(d x)<+\infty$ (if $M=\mathbb{R}$ we set $L^{p}(H, \mu ; \mathbb{R})=L^{p}(H, \mu)$ ). We will also use the notation $L^{p}(\mu)$ instead of $L^{p}(H, \mu, M)$ when no confusion may arise.

The semigroup $R_{t}$ can be uniquely extended to a strongly continuous semigroup of contractions on $L^{p}(H, \mu), p \geq 1$, which we still denote by $R_{t}$, whereas we denote by $L_{p}$ (or $L$ when no confusion may arise) its infinitesimal generator, which is defined on smooth functions $f$ as

$$
L f(x)=\frac{1}{2} \operatorname{Tr}\left(D^{2} f(x)\right)+\langle A x, D f(x)\rangle,
$$

where $D f(x)$ and $D^{2} f(x)$ denote, respectively, the first and second Fréchet derivatives of $f$ at $x \in H$. For real Banach spaces $F$ and $G$ we denote by $C_{b}^{k}(F, G), k \geq 1$, the Banach space of all functions $f: F \rightarrow G$ which are bounded and Fréchet differentiable on $F$ up to order $k \geq 1$ with all derivatives bounded and continuous on $H$. We also set $C_{b}^{k}(F, \mathbb{R})=C_{b}^{k}(F)$.

Following [7], for any $f \in B_{b}(H)$ and any $t>0$, one has $R_{t} f \in C_{b}^{\infty}(H)=$ $\cap_{k \geq 1} C_{b}^{k}(H)$. Moreover,

$$
\left\langle D R_{t} f(x), k\right\rangle=\int_{H}\left\langle\Lambda_{t} k, Q_{t}^{-\frac{1}{2}} y\right\rangle f\left(e^{t A} x+y\right) N\left(0, Q_{t}\right)(d y), \quad k \in H, x \in H,
$$

where $Q_{t}$ is as defined in (15),

$$
\Lambda_{t}=Q_{t}^{-1 / 2} e^{t A}=\sqrt{2}(-A)^{1 / 2} e^{t A}\left(I-e^{2 t A}\right)^{-1 / 2}
$$


and $y \mapsto\left\langle\Lambda_{t} k, Q_{t}^{-\frac{1}{2}} y\right\rangle$ is a centered Gaussian random variable under $\mu_{t}=N\left(0, Q_{t}\right)$ with variance $\left|\Lambda_{t} k\right|^{2}$, for any $t>0$ (cf. Theorem 6.2.2 in [6]). Since

$$
\Lambda_{t} e_{k}=\sqrt{2}\left(\lambda_{k}\right)^{1 / 2} e^{-t \lambda_{k}}\left(1-e^{-2 t \lambda_{k}}\right)^{-1 / 2} e_{k}, \quad k \geq 1,
$$

we see that there exists $C_{0}^{\prime}>0$ such that $\left\|\Lambda_{t}\right\| \leq C_{0}^{\prime} t^{-\frac{1}{2}}$.

In the sequel $\|\cdot\|$ denotes the Hilbert-Schmidt norm; on the other hand $\|\cdot\|_{\mathcal{L}}$ indicates the operator norm. By (17) we deduce, for any $\varphi \in B_{b}(H)$,

$$
\sup _{x \in H}\left|D R_{t} \varphi(x)\right|=\left\|D R_{t} \varphi\right\|_{0} \leq C_{0} t^{-\frac{1}{2}}\|\varphi\|_{0}, \quad t>0,
$$

which by taking the Laplace transform yields

$$
\left\|D\left(\lambda-L_{2}\right)^{-1} \varphi\right\|_{0} \leq \frac{C_{0}}{\lambda^{\frac{1}{2}}}\|\varphi\|_{0}, \quad \lambda>0
$$

Similarly, we find

$$
\left\|D R_{t} \varphi\right\|_{L^{2}(\mu)} \leq C_{0} t^{-\frac{1}{2}}\|\varphi\|_{L^{2}(\mu)}
$$

and

$$
\left\|D\left(\lambda-L_{2}\right)^{-1} \varphi\right\|_{L^{2}(\mu)} \leq \frac{C_{0}}{\lambda^{\frac{1}{2}}}\|\varphi\|_{L^{2}(\mu)} .
$$

Recall that the Sobolev space $W^{2, p}(H, \mu), p \geq 1$, is defined in Section 3 of [3] as the completion of a suitable set of smooth functions endowed with the Sobolev norm (cf. also Section 9.2 in [6] for the case $p=2$ and [24]). Under our above hypotheses, the following result is proved in Section 10.2.1 of [7].

Theorem 3. Let $\lambda>0, f \in L^{2}(H, \mu)$ and let $v \in D\left(L_{2}\right)$ be the solution of the equation

$$
\lambda v-L_{2} v=f .
$$

Then $v \in W^{2,2}(H, \mu),(-A)^{1 / 2} D v \in L^{2}(H, \mu ; H)$ and, moreover, there exists a constant $C(\lambda)$ such that

$$
\|v\|_{L^{2}(\mu)}+\left(\int_{H}\left\|D^{2} v(x)\right\|^{2} \mu(d x)\right)^{1 / 2}+\left\|(-A)^{1 / 2} D v\right\|_{L^{2}(\mu)} \leq C\|f\|_{L^{2}(\mu)} .
$$

The following extension to $L^{p}(\mu), p>1$, can be found in Section 3 of [3] (see also [2] and [20]).

Theorem 4. Let $\lambda>0, f \in L^{p}(H, \mu)$ and let $v \in D\left(L_{p}\right)$ be the solution of the equation

$$
\lambda v-L_{p} v=f .
$$

Then $v \in W^{2, p}(H, \mu),(-A)^{1 / 2} D v \in L^{p}(H, \mu ; H)$ and there exists a constant $C=$ $C(\lambda, p)$ such that

$$
\|v\|_{L^{p}(\mu)}+\left(\int_{H}\left\|D^{2} v(x)\right\|^{p} \mu(d x)\right)^{1 / p}+\left\|(-A)^{1 / 2} D v\right\|_{L^{p}(\mu)} \leq C\|f\|_{L^{p}(\mu)} .
$$




\section{Analytic results and an Itô type formula \\ 2.1 Existence and uniqueness for the Kolmogorov equation when $B$ is bounded}

Here we consider the equation

$$
\lambda u-L_{2} u-\langle B, D u\rangle=f,
$$

where $\lambda>0, f \in B_{b}(H)$ and $B \in B_{b}(H, H)$ (i.e., $B: H \rightarrow H$ is Borel and bounded).

Remark 5. Since the corresponding Dirichlet form

$$
\mathcal{E}(f, g):=\int_{H}\langle D f, D g\rangle d \mu-\int_{H}\langle B, D f\rangle g d \mu+\lambda \int_{H} f g d \mu,
$$

$f, g \in W^{1,2}(\mu)$, is weakly sectorial for $\lambda$ big enough, it follows by Chap. I and Subsection 3e in Chap. II of [19] that (20) has a unique solution in $D\left(L_{2}\right)$. However, we need more regularity for $u$.

We recall a result from [5].

Proposition 6. Let $\lambda \geq \lambda_{0}$, where

$$
\lambda_{0}:=4\|B\|_{0}^{2} C_{0}^{2} .
$$

Then there is a unique solution $u \in D\left(L_{2}\right)$ of (201) given by

$$
u=u_{\lambda}=\left(\lambda-L_{2}\right)^{-1}\left(I-T_{\lambda}\right)^{-1} f,
$$

where

$$
T_{\lambda} g:=\left\langle B, D\left(\lambda-L_{2}\right)^{-1} g\right\rangle .
$$

Moreover, $u \in C_{b}^{1}(H)$ with

$$
\|u\|_{0} \leq 2\|f\|_{0}, \quad\|D u\|_{0} \leq \frac{2 C_{1,0}}{\lambda^{\frac{1}{2}}}\|f\|_{0},
$$

and, for any $p \geq 2, u \in W^{2, p}(H, \mu)$ and, for some $c=c\left(\lambda, p,\|B\|_{0}\right)$,

$$
\int_{H}\left\|D^{2} u(x)\right\|^{p} \mu(d x) \leq c \int_{H}|f(x)|^{p} \mu(d x) .
$$




\subsection{Approximations}

We are given two sequences $\left(f_{n}\right) \subset B_{b}(H)$ and $\left(B_{n}\right) \subset B_{b}(H, H)$ and a constant $M>0$ such that

$$
\begin{aligned}
& \text { (i) } f_{n}(x) \rightarrow f(x), \quad B_{n}(x) \rightarrow B(x) \quad \mu \text {-a.e.. } \\
& \text { (ii) }\left\|f_{n}\right\|_{0} \leq M, \quad\left\|B_{n}\right\|_{0} \leq M, \quad n \geq 1 .
\end{aligned}
$$

The following result has been proved in [5].

Proposition 7. Let $\lambda \geq \lambda_{0}$, where $\lambda_{0}$ is given in (21). Then the equation

$$
\lambda u_{n}-L u_{n}-\left\langle B_{n}, D u_{n}\right\rangle=f_{n},
$$

has a unique solution $u_{n} \in C_{b}^{1}(H) \cap D\left(L_{2}\right)$ which is given by

$$
u_{n}=(\lambda-L)^{-1}\left(I-T_{n, \lambda}\right)^{-1} f_{n},
$$

where $T_{n, \lambda} \varphi:=\left\langle B_{n}, D\left(\lambda-L_{2}\right)^{-1} \varphi\right\rangle$. Moreover,

$$
\left\|u_{n}\right\|_{0} \leq 2 M, \quad\left\|D u_{n}\right\|_{0} \leq \frac{2 C_{1,0}}{\lambda^{\frac{1}{2}}} M, \quad n \geq 1
$$

Finally, we have $u_{n} \rightarrow u$, and $D u_{n} \rightarrow D u$, in $L^{2}(\mu)$, where $u$ is the solution to (20).

Next we prove a new result. The idea behind the result is that if $\left(f_{n}\right)$ satisfies (22) then, for any $x \in H$ (not only $\mu$-a.e.), $t>0$,

$$
R_{t} f_{n}(x) \rightarrow R_{t} f(x)
$$

as $n \rightarrow \infty$, due to the fact that, for any $x \in H$, the law of the OU process $Z(t, x)$ at time $t>0$ is absolutely continuous with respect to $\mu$.

Lemma 8. Consider the situation of Proposition 7 . Then we have:

$$
u_{n}(x) \rightarrow u(x), \quad D u_{n}(x) \rightarrow D u(x),
$$

for any $x \in H$.

Proof. By a standard argument, possibly passing to a subsequence, we may assume that $D u_{n}(x) \rightarrow D u(x), \mu$-a.e.. It follows that for any $x, \mu$-a.e.,

$$
f_{n}(x)+\left\langle B_{n}(x), D u_{n}(x)\right\rangle \rightarrow f(x)+\langle B(x), D u(x)\rangle,
$$

as $n \rightarrow \infty$. We write, for any $\lambda \geq \lambda_{0}, x \in H$,

$$
u_{n}(x)=\int_{0}^{\infty} e^{-\lambda t} R_{t}\left(f_{n}+\left\langle B_{n}, D u_{n}\right\rangle\right)(x) d t .
$$


By the argument used in (24) we can apply the dominated convergence theorem and obtain that $u_{n}(x) \rightarrow u(x)$, as $n \rightarrow \infty, x \in H$.

Concerning $D u_{n}$, using (19), we obtain, for any $x \in H, h \in H$,

$$
\left\langle D u_{n}(x), h\right\rangle=\int_{0}^{\infty} e^{-\lambda t}\left\langle D R_{t}\left(f_{n}+\left\langle B_{n}, D u_{n}\right\rangle\right)(x), h\right\rangle d t .
$$

Setting $g_{n}=f_{n}+\left\langle B_{n}, D u_{n}\right\rangle, g=f+\langle B, D u\rangle$, note that

$$
\left\langle D R_{t} g_{n}(x), e_{k}\right\rangle=\int_{H}\left\langle\Lambda_{t} e_{k}, Q_{t}^{-\frac{1}{2}} y\right\rangle g_{n}\left(e^{t A} x+y\right) N\left(0, Q_{t}\right)(d y), \quad k \geq 1, x \in H, t>0 .
$$

It follows that

$$
\left|\left\langle D R_{t}\left(g_{n}-g\right)(x), e_{k}\right\rangle\right|^{2} \leq\left|\Lambda_{t} e_{k}\right|^{2} \int_{H}\left|g\left(e^{t A} x+y\right)-g_{n}\left(e^{t A} x+y\right)\right|^{2} N\left(0, Q_{t}\right)(d y),
$$

and so

$$
\left|D R_{t}\left(g_{n}-g\right)(x)\right|^{2} \leq\left\|\Lambda_{t}\right\|^{2} \int_{H}\left|g\left(e^{t A} x+y\right)-g_{n}\left(e^{t A} x+y\right)\right|^{2} N\left(0, Q_{t}\right)(d y) .
$$

Now we get $\left|D R_{t}\left(g_{n}-g\right)(x)\right|^{2} \rightarrow 0$ as $n \rightarrow \infty$, for any $x \in H, t>0$, by the same argument used in (24).

Using again the dominated convergence theorem we obtain easily that $D u_{n}(x) \rightarrow$ $D u(x)$, as $n \rightarrow \infty$, for any $x \in H$.

\subsection{Modified mild formulation}

Recall the notation

$$
B_{N}=B 1_{B(0, N)}, \quad N \geq 1
$$

where $B(0, N)$ is the open ball of radius $N$ (hence $B_{N} \in B_{b}(H, H), N \geq 1$ ).

For any $i \in \mathbb{N}$ we denote the $i^{\text {th }}$ component of $B$ by $B^{(i)}$, i.e.,

$$
B^{(i)}(x):=\left\langle B(x), e_{i}\right\rangle, \quad x \in H
$$

Then for $\lambda \geq 4\left\|B_{N}\right\|_{0}^{2} C_{0}^{2}$ we consider the solution $u_{N}^{(i)}$ of the equation

$$
\lambda u_{N}^{(i)}-L u_{N}^{(i)}-\left\langle B_{N}, D u_{N}^{(i)}\right\rangle=B_{N}^{(i)}, \quad \mu \text {-a.e. }
$$

We recall that by Proposition 6, $u_{N}^{(i)} \in C_{b}^{1}(H)$ and, for any $p \geq 2, u_{N}^{(i)} \in W^{2, p}(H, \mu)$.

The next result is a kind of "local version" of Theorem 7 in [5]. In contrast to Theorem 1 the result holds for any initial condition $x \in H$. 
Remark 9. Compared with the proof of Theorem 7 in [5], here we will use Lemma 8 which allows to simplify some arguments of [5] and it is also needed to justify the approximation procedure (see in particular (41)). We also mention that differently with respect to [5] in Step 3 of the proof we need to construct a suitable auxiliary process $\hat{X}^{N}=\left(\hat{X}_{t}^{N}\right)$ (see (43) ) in order to apply the Girsanov theorem and get the assertion.

Theorem 10. Let $X=\left(X_{t}\right)$ be a weak mild solution of equation (1) defined on some filtered probability space with a cylindrical $\left(\mathcal{F}_{t}\right)$-Wiener process $W$. Consider the stopping time

$$
\tau_{N}^{X}=\inf \left\{t \geq 0: X_{t} \notin B(0, N)\right\}
$$

Let $u_{N}^{(i)}$ be the solution of (27) and set $X_{t}^{(i)}=\left\langle X_{t}, e_{i}\right\rangle$. For any $t>0$ we have $\mathbb{P}$-a.s. on the event $\left\{t \leq \tau_{N}^{X}\right\}$

$$
\begin{aligned}
X_{t}^{(i)}= & e^{-\lambda_{i} t}\left(\left\langle x, e_{i}\right\rangle+u_{N}^{(i)}(x)\right)-u_{N}^{(i)}\left(X_{t}\right) \\
& +\left(\lambda+\lambda_{i}\right) \int_{0}^{t} e^{-\lambda_{i}(t-s)} u_{N}^{(i)}\left(X_{s}\right) d s \\
+ & \int_{0}^{t} e^{-\lambda_{i}(t-s)}\left(d\left\langle W_{s}, e_{i}\right\rangle+\left\langle D u_{N}^{(i)}\left(X_{s}\right), d W_{s}\right\rangle\right) .
\end{aligned}
$$

Proof. We fix $t>0, N \geq 1$ and $i \geq 1$.

Step 1 Approximation of $B_{N}$ and $u_{N}$.

Set

$$
B_{N, n}(x)=\int_{H} B_{N}\left(e^{\frac{1}{n} A} x+y\right) N\left(0, Q_{\frac{1}{n}}\right)(d y), \quad x \in H .
$$

Then $B_{N, n}$ is of $C^{\infty}$ class and all its derivatives are bounded. Moreover, $\left\|B_{N, n}\right\|_{0} \leq$ $\left\|B_{N}\right\|_{0}, n \geq 1$. It is easy to see that, possibly passing to a subsequence,

$$
B_{N, n} \rightarrow B_{N}, \quad \mu-\text { a.e.. }
$$

(indeed $B_{N, n} \rightarrow B_{N}$ in $L^{2}(H, \mu ; H)$; this result can be first checked for continuous and bounded $B)$. Now we denote by $u_{N, n}^{(i)}$ the solution of the equation

$$
\lambda u_{N, n}^{(i)}-L u_{N, n}^{(i)}-\left\langle B_{N, n}, D u_{N, n}^{(i)}\right\rangle=B_{N, n}^{(i)}
$$

where $B_{N, n}^{(i)}=\left\langle B_{N, n}, e_{i}\right\rangle$. By Lemma 8 we have, possibly passing to a subsequence, for any $x \in H$,

$$
\begin{array}{r}
\lim _{n \rightarrow \infty} u_{N, n}^{(i)}(x)=u_{N}^{(i)}(x), \quad \lim _{n \rightarrow \infty} D u_{N, n}^{(i)}(x)=D u_{N}^{(i)}(x), \\
\sup _{n \geq 1}\left\|u_{N, n}^{(i)}\right\|_{C_{b}^{1}(H)}=C_{i, N}<\infty
\end{array}
$$

where $u_{N}^{(i)}$ is the solution of (27). 
Step 2 Approximation of $X_{t}$.

For any $m \geq i$ we set $X_{m}=\left(X_{m, t}\right), X_{m, t}:=\pi_{m} X_{t}$, where $\pi_{m}=\sum_{j=1}^{m} e_{j} \otimes e_{j}$. Then we have

$$
X_{m, t}=\pi_{m} x+\int_{0}^{t} A_{m} X_{s} d s+\int_{0}^{t} \pi_{m} B\left(X_{s}\right) d s+\pi_{m} W_{t},
$$

where $A_{m}=\pi_{m} A$.

Now we denote by $u_{N, n, m}^{(i)}$ the solution of the equation

$$
\lambda u_{N, n, m}^{(i)}-L u_{N, n, m}^{(i)}-\left\langle\pi_{m} B_{N, n} \circ \pi_{m}, D u_{N, n, m}^{(i)}\right\rangle=B_{N, n}^{(i)} \circ \pi_{m},
$$

where $\left(B_{N, n} \circ \pi_{m}\right)(x)=B_{N, n}\left(\pi_{m} x\right), x \in H$. Since only a finite number of variables is involved, we have, equivalently,

$$
\lambda u_{N, n, m}^{(i)}-L^{(m)} u_{N, n, m}^{(i)}-\left\langle\pi_{m} B_{N, n} \circ \pi_{m}, D u_{N, n, m}^{(i)}\right\rangle=B_{N, n}^{(i)} \circ \pi_{m},
$$

with

$$
L^{(m)} \varphi(x)=\frac{1}{2} \operatorname{Tr}\left[\pi_{m} D^{2} \varphi(x)\right]+\left\langle A_{m} x, D \varphi(x)\right\rangle .
$$

Moreover, since $u_{N, n, m}^{(i)}$ depends only on the first $m$ variables, we have

$$
u_{N, n, m}^{(i)}\left(\pi_{m} y\right)=u_{N, n, m}^{(i)}(y), \quad y \in H .
$$

Applying the finite-dimensional Itô formula to $u_{N, n, m}^{(i)}\left(X_{m, t}\right)=u_{N, n, m}^{(i)}\left(X_{t}\right)$ with the stopping time $\tau_{N}^{X}$ yields

$$
\begin{gathered}
u_{N, n, m}^{(i)}\left(X_{\left.m, t \wedge \tau_{N}^{X}\right)}\right)-u_{N, n, m}^{(i)}\left(\pi_{m} x\right)= \\
\int_{0}^{t \wedge \tau_{N}^{X}}\left(\frac{1}{2} \operatorname{Tr}\left[D^{2} u_{N, n, m}^{(i)}\left(X_{m, s}\right)\right]+\left\langle D u_{N, n, m}^{(i)}\left(X_{m, s}\right), A_{m} X_{s}+\pi_{m} B\left(X_{s}\right)\right\rangle\right) d s \\
+\int_{0}^{t \wedge \tau_{N}^{X}}\left\langle D u_{n, m}^{(i)}\left(X_{m, s}\right), \pi_{m} d W_{s}\right\rangle .
\end{gathered}
$$

On the other hand, by (34) we have

$$
\begin{aligned}
& \lambda u_{N, n, m}^{(i)}\left(X_{m, t}\right)-\frac{1}{2} \operatorname{Tr}\left[D^{2} u_{N, n, m}^{(i)}\left(X_{m, t}\right)\right] \\
& -\left\langle D u_{N, n, m}^{(i)}\left(X_{m, t}\right), A_{m} X_{m, t}+\pi_{m} B_{N, n}\left(X_{m, t}\right)\right\rangle=B_{N, n}^{(i)}\left(X_{m, t}\right) .
\end{aligned}
$$

Let us fix $r \in] 0, t]$. This will be useful in Step 3 of the proof to apply the Girsanov theorem (see in particular (45)).

Comparing with (37) and using (36) we find

$$
u_{N, n, m}^{(i)}\left(X_{t \wedge \tau_{N}^{X}}\right)-u_{N, n, m}^{(i)}\left(X_{r \wedge \tau_{N}^{X}}\right)
$$




$$
\begin{gathered}
=\lambda \int_{r \wedge \tau_{N}^{X}}^{t \wedge \tau_{N}^{X}} u_{N, n, m}^{(i)}\left(X_{s}\right) d s-\int_{r \wedge \tau_{N}^{X}}^{t \wedge \tau_{N}^{X}} B_{N, n}^{(i)}\left(X_{m, s}\right) d s \\
+\int_{r \wedge \tau_{N}^{X}}^{t \wedge \tau_{N}^{X}}\left\langle D u_{N, n, m}^{(i)}\left(X_{s}\right), \pi_{m}\left(B\left(X_{s}\right)-B_{N, n}\left(X_{m, s}\right)\right)\right\rangle d s+\int_{r \wedge \tau_{N}^{X}}^{t \wedge \tau_{N}^{X}}\left\langle D u_{N, n, m}^{(i)}\left(X_{s}\right), d W_{s}\right\rangle .
\end{gathered}
$$

Possibly passing to a subsequence, and taking the limit in probability as $m \rightarrow \infty$ (with respect to $\mathbb{P}$ ), we arrive at

$$
\begin{gathered}
u_{N, n}^{(i)}\left(X_{t \wedge \tau_{N}^{X}}\right)-u_{N, n}^{(i)}\left(X_{r \wedge \tau_{N}^{X}}\right) \\
=\lambda \int_{r \wedge \tau_{N}^{X}}^{t \wedge \tau_{N}^{X}} u_{N, n}^{(i)}\left(X_{s}\right) d s-\int_{r \wedge \tau_{N}^{X}}^{t \wedge \tau_{N}^{X}} B_{N, n}^{(i)}\left(X_{s}\right) d s \\
+\int_{r \wedge \tau_{N}^{X}}^{t \wedge \tau_{N}^{X}}\left\langle D u_{N, n}^{(i)}\left(X_{s}\right),\left(B\left(X_{s}\right)-B_{N, n}\left(X_{s}\right)\right)\right\rangle d s+\int_{r \wedge \tau_{N}^{X}}^{t \wedge \tau_{N}^{X}}\left\langle D u_{N, n}^{(i)}\left(X_{s}\right), d W_{s}\right\rangle .
\end{gathered}
$$

Let us justify this assertion.

First note that in equation (34) we have the drift term $\pi_{m} B_{N, n} \circ \pi_{m}$ which converges pointwise to $B_{N, n}$ and $B_{N, n}^{(i)} \circ \pi_{m}$ which converges pointwise to $B_{N, n}^{(i)}$ as $m \rightarrow \infty$. Since such functions are also uniformly bounded, we can apply Proposition 7 and Lemma 8 and obtain that, possibly passing to a subsequence (recall that $n$ is fixed),

$$
\begin{aligned}
\lim _{m \rightarrow \infty} u_{N, n, m}^{(i)}(x)= & u_{N, n}^{(i)}(x), \lim _{m \rightarrow \infty} D u_{N, n, m}^{(i)}(x)=D u_{N, n}^{(i)}(x), \quad x \in H, \\
& \sup _{m \geq 1}\left\|u_{N, n, m}^{(i)}\right\|_{C_{b}^{1}(H)}=C_{i}^{N}<\infty .
\end{aligned}
$$

We only consider convergence of the two most involved terms in (38).

We first treat convergence in $L^{2}(\Omega)$ of the stochastic integral. Recall that

$$
\int_{0}^{t \wedge \tau_{N}^{X}}\left\langle D u_{N, n}^{(i)}\left(X_{s}\right), d W_{s}\right\rangle=\int_{0}^{t} 1_{\left\{s \leq t \wedge \tau_{N}^{X}\right\}}\left\langle D u_{N, n}^{(i)}\left(X_{s}\right), d W_{s}\right\rangle
$$

by the isometry formula and (40) we get

$$
\mathbb{E}\left|\int_{0}^{t \wedge \tau_{N}^{X}}\left\langle D u_{N, n, m}^{(i)}\left(X_{s}\right)-D u_{N, n}^{(i)}\left(X_{s}\right), d W_{s}\right\rangle\right|^{2} \rightarrow 0
$$

as $m \rightarrow \infty$. Note that we have used that $\lim _{m \rightarrow \infty} D u_{N, n, m}^{(i)}(x)=D u_{N, n}^{(i)}(x)$, for any $x \in H$ (not only for $\mu$-a.e $x \in H$ ). In a similar way we get

$$
\mathbb{E}\left|\int_{0}^{r \wedge \tau_{N}^{X}}\left\langle D u_{N, n, m}^{(i)}\left(X_{s}\right)-D u_{N, n}^{(i)}\left(X_{s}\right), d W_{s}\right\rangle\right|^{2} \rightarrow 0
$$

as $m \rightarrow \infty$. 
This shows that $\int_{r \wedge \tau_{N}^{X}}^{t \wedge \tau_{N}^{X}}\left\langle D u_{N, n, m}^{(i)}\left(X_{s}\right), d W_{s}\right\rangle$ converges to $\int_{r \wedge \tau_{N}^{X}}^{t \wedge \tau^{X}}\left\langle D u_{N, n}^{(i)}\left(X_{s}\right), d W_{s}\right\rangle$ in $L^{2}(\Omega)$ as $m \rightarrow \infty$. To show that, $\mathbb{P}$-a.s.,

$$
\begin{aligned}
\lim _{m \rightarrow \infty} & \int_{r \wedge \tau_{N}^{X}}^{t \wedge \tau_{N}^{X}} \mid\left\langle D u_{N, n, m}^{(i)}\left(X_{s}\right), \pi_{m}\left(B\left(X_{s}\right)-B_{N, n}\left(X_{m, s}\right)\right)\right\rangle \\
& -\left\langle D u_{N, n}^{(i)}\left(X_{s}\right),\left(B\left(X_{s}\right)-B_{N, n}\left(X_{s}\right)\right)\right\rangle \mid d s=0
\end{aligned}
$$

it is enough to prove that $\lim _{m \rightarrow \infty} H_{m}+K_{m}=0$, where

$$
H_{m}=\int_{0}^{t \wedge \tau_{N}^{X}}\left|\left\langle D u_{N, n, m}^{(i)}\left(X_{s}\right)-D u_{N, n}^{(i)}\left(X_{s}\right), \pi_{m}\left(B\left(X_{s}\right)-B_{N, n}\left(X_{m, s}\right)\right)\right\rangle\right| d s
$$

and

$$
K_{m}=\int_{0}^{t \wedge \tau_{N}^{X}}\left|\left\langle D u_{N, n}^{(i)}\left(X_{s}\right),\left[\pi_{m} B\left(X_{s}\right)-B\left(X_{s}\right)\right]+\left[B_{N, n}\left(X_{s}\right)-\pi_{m} B_{N, n}\left(X_{m, s}\right)\right]\right\rangle\right| d s .
$$

By using (40) we easily get the assertion.

Step 3 A convergence result involving stopping times.

In order to pass to the limit in probability as $n \rightarrow \infty$ in (39) we recall formula (32) and argue as before. The only difficult term is

$$
\begin{gathered}
\int_{r \wedge \tau_{N}^{X}}^{t \wedge \tau_{N}^{X}}\left\langle D u_{N, n}^{(i)}\left(X_{s}\right),\left(B\left(X_{s}\right)-B_{N, n}\left(X_{s}\right)\right)\right\rangle d s=J_{n}+I_{n}, \\
\text { where } J_{n}=\int_{r \wedge \tau_{N}^{X}}^{t \wedge \tau_{N}^{X}}\left\langle D u_{N, n}^{(i)}\left(X_{s}\right)-D u_{N}^{(i)}\left(X_{s}\right),\left(B\left(X_{s}\right)-B_{N, n}\left(X_{s}\right)\right)\right\rangle d s, \\
I_{n}=\int_{r \wedge \tau_{N}^{X}}^{t \wedge \tau_{N}^{X}}\left\langle D u_{N}^{(i)}\left(X_{s}\right),\left(B_{N}\left(X_{s}\right)-B_{N, n}\left(X_{s}\right)\right)\right\rangle d s
\end{gathered}
$$

(using that $s \leq t \wedge \tau_{N}^{X}$ ). As for $J_{n}$ we have

$$
J_{n}=\int_{r \wedge \tau_{N}^{X}}^{t \wedge \tau_{N}^{X}}\left\langle D u_{N, n}^{(i)}\left(X_{s}\right)-D u_{N}^{(i)}\left(X_{s}\right),\left(B_{N}\left(X_{s}\right)-B_{N, n}\left(X_{s}\right)\right)\right\rangle d s
$$

and so $\left|J_{n}\right| \leq 2\left\|B_{N}\right\|_{0} \int_{0}^{t}\left|D u_{N, n}^{(i)}\left(X_{s}\right)-D u_{N}^{(i)}\left(X_{s}\right)\right| d s \rightarrow 0, \mathbb{P}$-a.s., as $n \rightarrow \infty$, by Lemma 8.

Let us consider $I_{n}$. We define an auxiliary process $\hat{X}^{N}=\left(\hat{X}_{t}^{N}\right)$ as follows:

$$
\hat{X}_{t}^{N}:=e^{t A} x+\int_{0}^{t} e^{(t-s) A} B_{N}\left(X_{s \wedge \tau_{N}^{X}}\right) d s+\int_{0}^{t} e^{(t-s) A} d W_{s}, \quad t \geq 0
$$


Note that $X_{s \wedge \tau_{N}^{X}}=\hat{X}_{s \wedge \tau_{N}^{X}}^{N}, s \geq 0$, so that

$$
\begin{aligned}
\left|I_{n}\right|= & \left|\int_{r \wedge \tau_{N}^{X}}^{t \wedge \tau_{N}^{X}}\left\langle D u_{N}^{(i)}\left(\hat{X}_{s}^{N}\right),\left(B_{N}\left(\hat{X}_{s}^{N}\right)-B_{N, n}\left(\hat{X}_{s}^{N}\right)\right)\right\rangle d s\right| \\
& \leq\left\|D^{(i)} u_{N}\right\|_{0} \int_{r}^{t}\left|B_{N}\left(\hat{X}_{s}^{N}\right)-B_{N, n}\left(\hat{X}_{s}^{N}\right)\right| d s .
\end{aligned}
$$

Now we use the Girsanov theorem (see e.g. Appendix in [5]). Let $T>0$. Since

$$
\hat{X}_{t}^{N}:=e^{t A} x+\int_{0}^{t} e^{(t-s) A} \hat{B}_{s}^{N} d s+\int_{0}^{t} e^{(t-s) A} d W_{s}, \quad t \geq 0
$$

where $\hat{B}_{s}^{N}=B_{N}\left(\hat{X}_{s \wedge \tau_{N}^{X}}^{N}\right), s \geq 0$, is an adapted and bounded process, we have that

$$
\tilde{W}_{t}^{N}:=W_{t}+\int_{0}^{t} \hat{B}_{s}^{N} d s
$$

is a cylindrical Wiener process on $\left(\Omega, \mathcal{F},\left(\mathcal{F}_{t}\right)_{t \in[0, T]}, \widetilde{\mathbb{P}}_{N}\right)$ where $\left.\frac{d \widetilde{\mathbb{P}}_{N}}{d \mathbb{P}}\right|_{\mathcal{F}_{T}}=\rho_{N}$,

$$
\rho_{N}=\exp \left(-\int_{0}^{T} \hat{B}_{s}^{N} d W_{s}-\frac{1}{2} \int_{0}^{T}\left|\hat{B}_{s}^{N}\right|^{2} d s\right)
$$

Hence $\hat{X}_{t}^{N}=e^{t A} x+\int_{0}^{t} e^{(t-s) A} d \tilde{W}_{s}^{N}$ is an OU process on $\left(\Omega, \mathcal{F},\left(\mathcal{F}_{t}\right)_{t \in[0, T]}, \tilde{\mathbb{P}}_{N}\right)$.

Moreover, we know that the law of $\left(\hat{X}_{t}^{N}\right)_{t \in[0, T]}$ on $C([0, T] ; H)$ is equivalent to the law of the OU process $Z(t, x)$ given in (16). In particular, all their transition probabilities are equivalent. Now under our assumptions the law of $Z(t, x)$ is equivalent to $\mu$ for all $t>0$ and $x \in H$ (see Theorem 11.3 in [6]).

Let us come back to (2.3). Using that the law $\pi_{t}^{N}(x, \cdot)$ of $\hat{X}_{t}^{N}$ is absolutely continuous with respect to $\mu$, we obtain

$$
\mathbb{E} \int_{r}^{t}\left|B_{N}\left(\hat{X}_{s}^{N}\right)-B_{N, n}\left(\hat{X}_{s}^{N}\right)\right| d s=\int_{r}^{t} d s \int_{H}\left|B_{N}(y)-B_{N, n}(y)\right| \frac{d \pi_{s}^{N}(x, \cdot)}{d \mu}(y) \mu(d y),
$$

which tends to 0 , as $n \rightarrow \infty$, by the dominated convergence theorem. Hence we have found that $I_{n} \rightarrow 0$ in $L^{1}(\Omega, \mathbb{P})$.

Up to now we have

$$
\begin{gathered}
u_{N}^{(i)}\left(X_{t \wedge \tau_{N}^{X}}\right)-u_{N}^{(i)}\left(X_{\left.r \wedge \tau_{N}^{X}\right)}\right) \\
=\lambda \int_{r \wedge \tau_{N}^{X}}^{t \wedge \tau_{N}^{X}} u_{N}^{(i)}\left(X_{s}\right) d s-\int_{r \wedge \tau_{N}^{X}}^{t \wedge \tau_{N}^{X}} B^{(i)}\left(X_{s}\right) d s+\int_{r \wedge \tau_{N}^{X}}^{t \wedge \tau_{N}^{X}}\left\langle D u_{N}^{(i)}\left(X_{s}\right), d W_{s}\right\rangle .
\end{gathered}
$$


Passing to the limit as $r \rightarrow 0^{+}$, since the trajectories of $X$ are continuous, we finally get

$$
\begin{gathered}
u_{N}^{(i)}\left(X_{\left.t \wedge \tau_{N}^{X}\right)}\right) u_{N}^{(i)}(x) \\
=\lambda \int_{0}^{t \wedge \tau_{N}^{X}} u_{N}^{(i)}\left(X_{s}\right) d s-\int_{0}^{t \wedge \tau_{N}^{X}} B^{(i)}\left(X_{s}\right) d s+\int_{0}^{t \wedge \tau_{N}^{X}}\left\langle D u_{N}^{(i)}\left(X_{s}\right), d W_{s}\right\rangle .
\end{gathered}
$$

Step 4 The final formula.

By (1) we deduce

$$
d X_{t}^{(i)}=-\lambda_{i} X_{t}^{(i)} d t+B^{(i)}\left(X_{t}\right) d t+d W_{t}^{(i)} .
$$

Inserting the expression for $B^{(i)}\left(X_{t}\right)$, which we get from this identity, into (46), we obtain

$$
\begin{gathered}
u_{N}^{(i)}\left(X_{t \wedge \tau_{N}^{X}}\right)-u_{N}^{(i)}(x) \\
=-X_{t \wedge \tau_{N}^{X}}^{i}+x^{i}+\lambda \int_{0}^{t \wedge \tau_{N}^{X}} u_{N}^{(i)}\left(X_{s}\right) d s-\lambda_{i} \int_{0}^{t \wedge \tau_{N}^{X}} X_{s}^{(i)} d s+\int_{0}^{t \wedge \tau_{N}^{X}}\left\langle D u_{N}^{(i)}\left(X_{s}\right), d W_{s}\right\rangle \\
+W_{t \wedge \tau_{N}^{X}}^{i} .
\end{gathered}
$$

By the variation of constants formula this is equivalent to

$$
\begin{gathered}
X_{t \wedge \tau_{N}^{X}}^{(i)}=e^{-\lambda_{i} t \wedge \tau_{N}^{X}}\left\langle x, e_{i}\right\rangle+\lambda \int_{0}^{t \wedge \tau_{N}^{X}} e^{-\lambda_{i}\left(t \wedge \tau_{N}^{X}-s\right)} u_{N}^{(i)}\left(X_{s}\right) d s \\
-\int_{0}^{t \wedge \tau_{N}^{X}} e^{-\lambda_{i}\left(t \wedge \tau_{N}^{X}-s\right)} d u_{N}^{(i)}\left(X_{s}\right)+e^{-\lambda_{i}\left(t \wedge \tau_{N}^{X}\right)} \int_{0}^{t \wedge \tau_{N}^{X}} e^{\lambda_{i} s}\left[d W_{s}^{(i)}+\left\langle D u_{N}^{(i)}\left(X_{s}\right), d W_{s}\right\rangle\right] .
\end{gathered}
$$

This identity yields (28) on $\left\{t \leq \tau_{N}^{X}\right\}$.

The next lemma is similar to Lemma 9 in [5] and shows that $u_{N}(x)=\sum_{k \geq 1} u_{N}^{(k)}(x) e_{k}$ (where $u_{N}^{(k)}$ is as in (27) ) is a well defined function which belongs to $C_{b}^{1}(H, H)$.

Lemma 11. For $\lambda$ sufficiently large, i.e., $\lambda \geq \tilde{\lambda}$, with $\tilde{\lambda}=\tilde{\lambda}\left(A,\left\|B_{N}\right\|_{0}\right)>0$ there exists a unique $u_{N}=u_{\lambda, N} \in C_{b}^{1}(H, H)$ which solves

$$
u_{N}(x)=\int_{0}^{\infty} e^{-\lambda t} R_{t}\left(D u_{N}(\cdot) B_{N}(\cdot)+B_{N}(\cdot)\right)(x) d t, \quad x \in H,
$$

where $R_{t}$ is the OU semigroup defined as in (14) and acting on H-valued functions. Moreover, we have the following assertions.

(i) For any $h \in H, D u_{N}(\cdot)[h] \in C_{b}(H, H)$ and $\left\|D u_{N}(\cdot)[h]\right\|_{0} \leq C_{0, \lambda, N}|h|$;

(ii) for any $k \geq 1,\left\langle u_{N}(\cdot), e_{k}\right\rangle=u_{N}^{(k)}$, where $u_{N}^{(k)}$ is the solution defined in (27);

(iii) There exists $c_{3}=c_{3}\left(A,\left\|B_{N}\right\|_{0}\right)>0$ such that, for any $\lambda \geq \tilde{\lambda}, u=u_{\lambda}$ satisfies

$$
\left\|D u_{N}\right\|_{0} \leq \frac{c_{3}}{\sqrt{\lambda}}
$$




\section{Proof of Theorem 1}

Let $X=\left(X_{t}\right)$ and $Y=\left(Y_{t}\right)$ be two weak mild solutions (see (8) and (9) ) defined on the same filtered probability space (solutions with respect to the same cylindrical Wiener process $W$ ) starting at $x \in H$.

In the first part of the proof we will adapt the proof of Theorem 1 in [5] by introducing additional stopping times. The main difference with respect to [5] will appear in Proposition 13 which is needed to finish the proof.

For the time being, $x$ is not specified. In Proposition 13 a restriction on $x$ will emerge.

Note that by our hypothesis

$$
B_{N}=B 1_{B(0, N)}=B^{\prime} 1_{B(0, N)}=B_{N}^{\prime} .
$$

It follows that Kolmogorov equation (27) written with respect to the truncated drift $B_{N}$ (with $B_{N}^{(i)}$ in the right-hand side) or with respect to $B_{N}^{\prime}$ (with $B_{N}^{\prime(i)}$ in the right-hand side) is the same and gives the same solution $u_{N, \lambda}^{(i)}$.

It follows that both $X$ and $Y$ satisfy (28) on the event $\left\{t \leq \tau_{N}^{X} \wedge \tau_{N}^{Y}\right\}$.

Now we consider

$$
u_{N}=u_{N, \lambda}: H \rightarrow H
$$

be such that $u_{N}(x)=\sum_{i \geq 1} u_{N}^{(i)}(x) e_{i}, x \in H$, where $u_{N}^{(i)}=u_{N, \lambda}^{(i)}$ solve (27) for some $\lambda$ large enough possibly depending on $N$.

Let us fix $T>0$. By (28), taking into account (48), we have, for $t \in\left[0, T \wedge \tau_{N}^{X} \wedge \tau_{N}^{Y}\right]$, P-a.s.,

$$
\begin{aligned}
X_{t}-Y_{t} & =u_{N}\left(Y_{t}\right)-u_{N}\left(X_{t}\right)+(\lambda-A) \int_{0}^{t} e^{(t-s) A}\left(u_{N}\left(X_{s}\right)-u_{N}\left(Y_{s}\right)\right) d s \\
& +\int_{0}^{t} e^{(t-s) A}\left(D u_{N}\left(X_{s}\right)-D u_{N}\left(Y_{s}\right)\right) d W_{s} .
\end{aligned}
$$

Here and in the sequel we will drop the $\lambda$-dependence of $u_{N}$ to simplify notation. However, at the end we will fix a value of $\lambda$ large enough. By (47) we may assume that $\left\|D u_{N}\right\|_{0} \leq 1 / 2$.

It follows that for $t \in\left[0, T \wedge \tau_{N}^{X} \wedge \tau_{N}^{Y}\right]$,

$$
\begin{aligned}
\left|X_{t}-Y_{t}\right| \leq \frac{1}{2}\left|X_{t}-Y_{t}\right|+\mid & (\lambda-A) \int_{0}^{t} e^{(t-s) A}\left(u_{N}\left(X_{s}\right)-u_{N}\left(Y_{s}\right)\right) d s \mid \\
& +\left|\int_{0}^{t} e^{(t-s) A}\left(D u_{N}\left(X_{s}\right)-D u_{N}\left(Y_{s}\right)\right) d W_{s}\right| .
\end{aligned}
$$

Let $\eta$ be a stopping time to be specified later and set

$$
\tau=\eta \wedge T \wedge \tau_{N}^{X} \wedge \tau_{N}^{Y} .
$$


Using that $1_{[0, \tau]}(t)=1_{[0, \tau]}(t) \cdot 1_{[0, \tau]}(s), 0 \leq s \leq t \leq T$, we have (cf. page 187 in [6])

$$
\begin{aligned}
& 1_{[0, \tau]}(t)\left|X_{t}-Y_{t}\right| \leq C 1_{[0, \tau]}(t)\left|(\lambda-A) \int_{0}^{t} e^{(t-s) A}\left(u_{N}\left(X_{s}\right)-u_{N}\left(Y_{s}\right)\right) d s\right| \\
& +C\left|1_{[0, \tau]}(t) \int_{0}^{t} e^{(t-s) A}\left(D u_{N}\left(X_{s}\right)-D u_{N}\left(Y_{s}\right)\right) 1_{[0, \tau]}(s) d W_{s}\right|,
\end{aligned}
$$

where by $C$ we denote any constant which may depend on the hypotheses on $A, B_{N}$ and $T$.

Setting $1_{[0, \tau]}(s) X_{s}=\tilde{X}_{s}$ and $1_{[0, \tau]}(s) Y_{s}=\tilde{Y}_{s}$, and, using the Burkholder-DavisGundy inequality with $q>2$ which will be determined below, we obtain (recall that $\|\cdot\|$ is the Hilbert-Schmidt norm, cf. Chapter 4 in [6]) with $C=C_{q}$,

$$
\begin{gathered}
\mathbb{E}\left[\left|\tilde{X}_{t}-\tilde{Y}_{t}\right|^{q}\right] \leq C \mathbb{E}\left[e^{\lambda q t}\left|(\lambda-A) \int_{0}^{t} e^{(t-s) A} e^{-\lambda s}\left(u_{N}\left(X_{s}\right)-u_{N}\left(Y_{s}\right)\right) 1_{[0, \tau]}(s) d s\right|^{q}\right] \\
+C \mathbb{E}\left[\left(\int_{0}^{t} 1_{[0, \tau]}(s)\left\|e^{(t-s) A}\left(D u_{N}\left(X_{s}\right)-D u_{N}\left(Y_{s}\right)\right)\right\|^{2} d s\right)^{q / 2}\right] .
\end{gathered}
$$

In the sequel we also consider a parameter $\theta>0$; moreover, $C_{\theta}$ will denote suitable positive constants such that $C_{\theta} \rightarrow 0$ as $\theta \rightarrow+\infty$. Similarly, $C(\lambda)$ will denote suitable constants (possibly depending on $N$ ) such that $C(\lambda) \rightarrow 0$ as $\lambda \rightarrow \infty$.

From the previous inequality we deduce, multiplying by $e^{-q \theta t}$, for any $\theta>0$,

$$
\begin{gathered}
\mathbb{E}\left[e^{-q \theta t}\left|\tilde{X}_{t}-\widetilde{Y}_{t}\right|^{q}\right] \\
\leq C \mathbb{E}\left[\left|(\lambda-A) \int_{0}^{t} e^{-\theta(t-s)} e^{(t-s) A}\left(u_{N}\left(X_{s}\right)-u_{N}\left(Y_{s}\right)\right) e^{-\theta s} 1_{[0, \tau]}(s) d s\right|^{q}\right] \\
+C \mathbb{E}\left[\left(\int_{0}^{t} e^{-2 \theta(t-s)}\left\|e^{(t-s) A}\left(D u_{N}\left(X_{s}\right)-D u_{N}\left(Y_{s}\right)\right)\right\|^{2} e^{-2 \theta s} 1_{[0, \tau]}(s) d s\right)^{q / 2}\right] .
\end{gathered}
$$

Now proceeding as in the proof of Theorem 7 of [5] we arrive at

$$
\begin{gathered}
\int_{0}^{T} \mathbb{E}\left[e^{-q \theta t}\left|\tilde{X}_{t}-\tilde{Y}_{t}\right|^{q}\right] d t \\
\leq C(\lambda) \int_{0}^{T} e^{-q \theta s} \mathbb{E}\left|\tilde{X}_{s}-\tilde{Y}_{s}\right|^{q} d s+\tilde{C}_{\theta} \mathbb{E}\left[\Lambda_{T} \cdot \int_{0}^{T} e^{-q \theta s}\left|\tilde{X}_{s}-\tilde{Y}_{s}\right|^{q} d s\right]
\end{gathered}
$$

provided that $q \in(4, \infty), \gamma=q / 2, \theta \geq \lambda$ and

$$
\begin{gathered}
\Lambda_{T}:=\int_{0}^{T} d t \int_{0}^{t} 1_{[0, \tau]}(s) d s \int_{0}^{1}\left(\sum_{n \geq 1} \frac{1}{\lambda_{n}^{1-\delta}}\left\|D^{2} u_{N}^{(n)}\left(Z_{s}^{r}\right)\right\|^{2}\right)^{\gamma} d r, \\
\text { where } Z_{t}^{r}=r X_{t}+(1-r) Y_{t},
\end{gathered}
$$

and $D^{2} u_{N}^{(n)}(x)$ is defined for $\mu$-a.e. $x \in H$. The existence of $D^{2} u_{N}^{(n)} \in L^{p}(\mu), p \geq 2$, follows from Proposition [6 applied to equation (27) (see also Lemma 23 in [5]). 
Since

$$
\Lambda_{T} \leq T \cdot \int_{0}^{T \wedge \tau} d s \int_{0}^{1}\left(\sum_{n \geq 1} \frac{1}{\lambda_{n}^{1-\delta}}\left\|D^{2} u_{N}^{(n)}\left(Z_{s}^{r}\right)\right\|^{2}\right)^{\gamma} d r
$$

it is natural to define, for any $R>0$, the stopping time

$$
\bar{\tau}_{R}^{x, N}=\inf \left\{t \geq 0: \int_{0}^{t} d s \int_{0}^{1}\left(\sum_{n \geq 1} \frac{1}{\lambda_{n}^{1-\delta}}\left\|D^{2} u_{N}^{(n)}\left(Z_{s}^{r}\right)\right\|^{2}\right)^{\gamma} d r \geq R\right\} \wedge T .
$$

Take $\eta=\bar{\tau}_{R}^{x, N}$ in the previous expressions so that

$$
\tau=\bar{\tau}_{R}^{x, N} \wedge \tau_{N}^{X} \wedge \tau_{N}^{Y}
$$

We get from (51)

$$
\begin{gathered}
\int_{0}^{T} e^{-q \theta t} \mathbb{E}\left|\tilde{X}_{t}-\tilde{Y}_{t}\right|^{q} d t \\
\leq C(\lambda) \int_{0}^{T} e^{-q \theta s} \mathbb{E}\left|\tilde{X}_{s}-\tilde{Y}_{s}\right|^{q} d s+\tilde{C}_{\theta} R \int_{0}^{T} e^{-q \theta s} \mathbb{E}\left|\tilde{X}_{s}-\tilde{Y}_{s}\right|^{q} d s .
\end{gathered}
$$

Now we fix $\lambda$ large enough such that $C(\lambda)<1 / 2$. For sufficiently large $\theta=\theta_{R} \geq \lambda$, depending on $R$ and $N$, we have $\tilde{C}_{\theta} R<1 / 2$ and so

$$
\mathbb{E}\left[\int_{0}^{T} e^{-q \theta_{R} t} 1_{[0, \tau]}(t)\left|X_{t}-Y_{t}\right|^{q} d t\right]=\mathbb{E}\left[\int_{0}^{\tau} e^{-q \theta_{R} t}\left|X_{t}-Y_{t}\right|^{q} d t\right]=0 .
$$

In other words, for every $R>0, N \geq 1, \mathbb{P}$-a.s., $X=Y$ on $\left[0, \bar{\tau}_{R}^{x, N} \wedge \tau_{N}^{X} \wedge \tau_{N}^{Y}\right]$ (identically in $t$, not only a.e. in $t$, since $X$ and $Y$ are continuous processes).

If we prove that

$$
\lim _{R \rightarrow \infty} \bar{\tau}_{R}^{x, N}=T \wedge \tau_{N}^{X} \wedge \tau_{N}^{Y}, \quad \mathbb{P}-\text { a.s. }
$$

then we obtain that $X=Y$ on $\left[0, T \wedge \tau_{N}^{X} \wedge \tau_{N}^{Y}\right]$ and this finishes the proof.

The crucial assertion (53) follows by the next proposition.

Remark 12. Assertion (53) is a "local version" of Proposition 10 in [5. Similarly to (43) also in the next proof we have to find an auxiliary process (see (54)) which allows to apply the Girsanov theorem.

Proposition 13. Let $N \geq 1$ and $T>0$ and suppose $X$ and $Y$ as in Theorem [1. For p-a.e. $x \in H$, we have $\mathbb{P}\left(S_{T \wedge \tau_{N}^{X} \wedge \tau_{N}^{Y}}^{x}<\infty\right)=1$, where

$$
S_{t}^{x}=S_{t}^{x, N}=\int_{0}^{t} \int_{0}^{1}\left(\sum_{n \geq 1} \frac{1}{\lambda_{n}^{1-\delta}}\left\|D^{2} u_{N}^{(n)}\left(Z_{s}^{r}\right)\right\|^{2}\right)^{\gamma} d r d s, \quad t \in[0, T],
$$

with $\gamma=q / 2\left(u_{N}(x)=\sum_{i \geq 1} u_{N}^{(i)}(x) e_{i}, x \in H\right.$, where $u_{N}^{(i)}=u_{N, \lambda}^{(i)}$ solves (27) $)$. 
Proof. To prove the assertion we will show that, for $\mu$-a.e. $x \in H$,

$$
\mathbb{E}\left[S_{T \wedge \tau_{N}^{X} \wedge \tau_{N}^{Y}}^{x}\right]<+\infty .
$$

In the first part of the proof, $x \in H$ is given, without restriction. Let us consider stopped processes

$$
X_{t}^{N}=X_{t \wedge \tau_{N}^{X} \wedge \tau_{N}^{Y}}, \quad Y_{t}^{N}=Y_{t \wedge \tau_{N}^{X} \wedge \tau_{N}^{Y}}
$$

and then we define an auxiliary process $\left(Z_{t}^{r, N}\right)_{t \in[0, T]}$ as follows

$$
Z_{t}^{r, N}:=e^{t A} x+\int_{0}^{t} e^{(t-s) A} \bar{B}_{s}^{r, N} d s+\int_{0}^{t} e^{(t-s) A} d W_{s}
$$

where (recall (10))

$$
\bar{B}_{s}^{r, N}:=\left[r B\left(X_{s}^{N}\right)+(1-r) B\left(Y_{s}^{N}\right)\right], \quad r \in[0,1], \quad s \in[0, T] .
$$

Comparing $Z^{r}$ (see (3i)) and $Z^{r, N}$ we see that $Z_{s \wedge \tau_{N}^{X} \wedge \tau_{N}^{Y}}^{r}=Z_{s \wedge \tau_{N}^{X} \wedge \tau_{N}^{Y}}^{r, N}, s \in[0, T], r \in[0,1]$. Hence we have to prove

$$
\mathbb{E}\left[S_{T \wedge \tau_{N}^{X} \wedge \tau_{N}^{Y}}^{x}\right]=\mathbb{E} \int_{0}^{T \wedge \tau_{N}^{X} \wedge \tau_{N}^{Y}} \int_{0}^{1}\left(\sum_{n \geq 1} \frac{1}{\lambda_{n}^{1-\delta}}\left\|D^{2} u_{N}^{(n)}\left(Z_{s}^{r, N}\right)\right\|^{2}\right)^{\gamma} d r d s<\infty .
$$

This follows if we can show that

$$
\mathbb{E} \int_{0}^{T} \int_{0}^{1}\left(\sum_{n \geq 1} \frac{1}{\lambda_{n}^{1-\delta}}\left\|D^{2} u_{N}^{(n)}\left(Z_{s}^{r, N}\right)\right\|^{2}\right)^{\gamma} d r d s<\infty
$$

We fix $N \geq 1$. To verify (55) we can follow the proof of Proposition 10 in [5]. We only indicate the small changes which are needed.

Define

$$
\rho_{r, N}=\exp \left(-\int_{0}^{t} \bar{B}_{s}^{r, N} d W_{s}-\frac{1}{2} \int_{0}^{t}\left|\bar{B}_{s}^{r, N}\right|^{2} d s\right) .
$$

We have, since $\left|\bar{B}_{s}^{r}\right| \leq\left\|B_{N}\right\|_{0}, r \in[0,1], s \geq 0, \mathbb{P}$-a.s.,

$$
\mathbb{E}\left[\exp \left(k \int_{0}^{T}\left|\bar{B}_{s}^{r, N}\right|^{2} d s\right)\right] \leq C_{k}<\infty,
$$

for all $k \in \mathbb{R}$, independently of $x$ and $r$, simply because $B_{N}$ is bounded. Hence an infinite dimensional version of Girsanov's Theorem with respect to a cylindrical Wiener process (the proof of which is included in the Appendix of [5]) applies and gives us that

$$
\tilde{W}_{t}^{N}:=W_{t}+\int_{0}^{t} \bar{B}_{s}^{r, N} d s
$$


is a cylindrical Wiener process on $\left(\Omega, \mathcal{F},\left(\mathcal{F}_{t}\right)_{t \in[0, T]}, \widetilde{\mathbb{P}}_{r, N}\right)$ where $\left.\frac{d \widetilde{\mathbb{P}}_{r, N}}{d \mathbb{P}}\right|_{\mathcal{F}_{T}}=\rho_{r, N}$. Hence

$$
Z_{t}^{r, N}=e^{t A} x+\int_{0}^{t} e^{(t-s) A} d \tilde{W}_{s}^{N}
$$

is an OU process on $\left(\Omega, \mathcal{F},\left(\mathcal{F}_{t}\right)_{t \in[0, T]}, \widetilde{\mathbb{P}}_{r, N}\right)$. Continuing as in the proof of Proposition 10 in [5] with $u^{(n)}$ replaced by $u_{N}^{(n)}, \rho_{r}$ replaced by $\rho_{r, N}, Z_{s}^{r}$ replaced by $Z_{s}^{r, N}, \bar{B}_{s}$ replaced by $\bar{B}_{s}^{r, N}$, we see that (55) holds if we prove that

$$
\int_{0}^{T} \mathbb{E}\left[\left(\sum_{n \geq 1} \frac{1}{\lambda_{n}^{1-\delta}}\left\|D^{2} u_{N}^{(n)}\left(e^{s A} x+W_{A}(s)\right)\right\|^{2}\right)^{2 \gamma}\right] d s<\infty
$$

where $W_{A}(t)=\int_{0}^{t} e^{(t-s) A} d W(s), \quad t \geq 0$. If $\mu_{s}^{x}$ denotes the law of $e^{s A} x+W_{A}(s)$, we have to prove that

$$
\int_{0}^{T} \int_{H}\left(\sum_{n \geq 1} \frac{1}{\lambda_{n}^{1-\delta}}\left\|D^{2} u_{N}^{(n)}(y)\right\|^{2}\right)^{2 \gamma} \mu_{s}^{x}(d y) d s<\infty .
$$

This can be checked as in the mentioned proof (see in particular Steps 3 and 4 in that proof) only for $\mu$-a.e. $x \in H$; one has to replace $B$ in the proof in [5] with our $B_{N}$.

Remark 14. As is easily checked in Theorem 1 the ball $B(0, N)$ can be replaced by any open bounded set in $H$.

Remark 15. According to Remark 11 in [5] our Theorem 1 provides an alternative approach to Veretennikov's uniqueness result in finite dimension. In this respect first note that Theorem 1 when $H=\mathbb{R}^{d}$ does not require to start from $\mu$-a.e. initial conditions $x$, but works for any initial $x \in \mathbb{R}^{d}$.

Note that in finite dimension an SDE like $d X_{t}=b\left(X_{t}\right) d t+d W_{t}$ with $b$ Borel and bounded is equivalent to $d X_{t}=-X_{t} d t+\left(b\left(X_{t}\right)+X_{t}\right) d t+d W_{t}$ which is in the form (11) with $A=-I$, and with a drift term $B(x)=b(x)+x$ which is completely covered by Theorem 1 ,

Recall that in this alternative approach to Veretennikov's result, basically the elliptic $L^{p}$-estimates with respect to Lebesgue measure used in [26] are replaced by elliptic $L^{p}(\mu)$-estimates.

\section{$4 \quad$ Existence of strong mild solutions}

Here we will use our Theorem 1 to prove existence of strong mild solutions when $B$ grows more than linearly. We will construct such solutions for $\mu$-a.e. initial $x \in H$.

According to Chapter 1 in [21] (see also [18]) if $x \in H$ we say that equation (11) has a (global) strong mild solution if, for every filtered probability space $\left(\Omega, \mathcal{F},\left(\mathcal{F}_{t}\right), \mathbb{P}\right)$ on 
which there is defined a cylindrical $\left(\mathcal{F}_{t}\right)$-Wiener process $W$ there exists an $H$-valued continuous $\left(\mathcal{F}_{t}\right)$-adapted process $X=\left(X_{t}\right)=\left(X_{t}\right)_{t \geq 0}$ such that

$$
\left(\Omega, \mathcal{F},\left(\mathcal{F}_{t}\right), \mathbb{P}, W, X\right)
$$

is a weak mild solution.

Theorem 16. Let us consider equation (10) and assume Hypothesis 1 and $B \in B_{b, l o c}(H, H)$. Moreover, suppose that there exist $C>0, p>0$, such that

$$
\langle B(y+z), y\rangle \leq C\left(|y|^{2}+e^{p|z|}+1\right), \quad y, z \in H
$$

Then, for $\mu$-a.e. $x \in H$ (where $\mu=N\left(0,-\frac{1}{2} A^{-1}\right)$ ), equation (1) has a strong mild solution. Moreover, this solution is pathwise unique.

Remark 17. Condition (59) is a bit stronger than the classical one: $\langle B(y), y\rangle \leq$ $C\left(|y|^{2}+1\right), y \in H$, which is usually imposed in finite dimension to have non-explosion for SDEs with additive noise. We can not use such condition. Indeed for a given mild solution $\left(X_{t}\right)$ we can not write the Itô formula for $\left|X_{t}\right|^{2}$ due to the fact that our noise is cylindrical.

To prove the result we will use our Theorem 1 together with a generalization of the Yamada-Watanabe theorem (see Theorem 2 in [21] and [18]) and some a-priori estimates on mild solutions (see Section 4.1).

Example 18. To introduce an example of a drift $\tilde{B}$ which satisfies the assumptions of Theorem [16, we first consider a measurable function $g: \mathbb{R} \rightarrow \mathbb{R}_{+}$such that $g(s)=0$ if $s \geq 0$ and $0 \leq g(s) \leq C e^{q|s|}, s<0$, for some $q>0$. It is easy to check that $g(s+r) r \leq C^{\prime}\left(1+|r|^{2}+e^{p|s|}\right), s, r \in \mathbb{R}$. We define $\tilde{B}: H \rightarrow H$,

$$
\tilde{B}(x)=\sum_{k \geq 1} \frac{g\left(x_{k}\right)}{k^{2}} e_{k}, \quad x \in H .
$$

It is not difficult to verify that $\tilde{B}$ satisfies the assumptions of the previous theorem. We can also add to our drift $\tilde{B}$ one of the singular drifts considered in Section 4 of [5]; we will still obtain an admissible drift for our theorem.

\subsection{An a-priori estimate}

Here we prove an a-priori estimate for mild solutions to (11) under condition (59). For this purpose let us consider the OU process

$$
Z_{t}=Z(t, x)=e^{t A} x+\int_{0}^{t} e^{(t-s) A} d W_{s}
$$


which under our hypotheses has a continuous $H$-valued version. It satisfies

$$
\left\langle Z_{t}, \varphi\right\rangle=\int_{0}^{t}\left\langle Z_{s}, A \varphi\right\rangle d s+\left\langle W_{t}, \varphi\right\rangle
$$

for all $\varphi \in D(A)$. By Proposition 18 in [5] we deduce, in particular, that for any $p>0$, $T>0$

$$
K_{T}:=\mathbb{E}\left[\sup _{t \in[0, T]} e^{p\left|Z_{t}\right|}\right]<\infty .
$$

Recall that under our hypotheses a weak mild solution to (11) can be defined, equivalently, as a tuple $\left(\Omega, \mathcal{F},\left(\mathcal{F}_{t}\right), \mathbb{P}, W, X\right)$, where $\left(\Omega, \mathcal{F},\left(\mathcal{F}_{t}\right), \mathbb{P}\right)$ is a filtered probability space on which there is defined a cylindrical $\left(\mathcal{F}_{t}\right)$-Wiener process $W$ and a continuous $\left(\mathcal{F}_{t}\right)$-adapted $H$-valued process $X=\left(X_{t}\right)=\left(X_{t}\right)_{t \geq 0}$ such that, $\mathbb{P}$-a.s.,

$$
\left\langle X_{t}, \varphi\right\rangle=\langle x, \varphi\rangle+\int_{0}^{t}\left(\left\langle X_{s}, A \varphi\right\rangle+\left\langle B\left(X_{s}\right), \varphi\right\rangle\right) d s+\left\langle W_{t}, \varphi\right\rangle, \quad t \geq 0,
$$

for all $\varphi \in D(A)$ (cf. Chapter 6 of [6]).

Theorem 19. Assume Hypothesis $1, B \in B_{b, l o c}(H, H)$ and condition (59). Let $X=$ $\left(X_{t}\right)_{t \geq 0}$ be a weak mild solution of equation (1) with $X_{0}=x \in H$.

There exists $C_{p}>0$ (possibly depending on $C$ and $p$ given in (59) ) such that

$$
\mathbb{E}\left[\sup _{t \in[0, T]}\left|X_{t}\right|^{2}\right] \leq e^{C_{p} T}\left(|x|^{2}+K_{T}+1\right), T>0 .
$$

Proof. The process $Y_{t}=X_{t}-Z_{t}$ satisfies

$$
\left\langle Y_{t}, \varphi\right\rangle=\langle x, \varphi\rangle+\int_{0}^{t}\left(\left\langle Y_{s}, A \varphi\right\rangle+\left\langle B\left(Y_{s}+Z_{s}\right), \varphi\right\rangle\right) d s
$$

for all $\varphi \in D(A)$, and it has continuous trajectories in $H$. Let us consider (61) with $\varphi=e_{k}($ see $(130))$ and set $Y_{t}^{(k)}=\left\langle Y_{t}, e_{k}\right\rangle$. Since $\frac{d Y_{t}^{(k)}}{d t} \cdot Y_{t}^{(k)} \leq B^{(k)}\left(Y_{t}+Z_{t}\right) Y_{t}^{(k)}$, we find

$$
\sum_{k \geq 1}\left\langle Y_{t}, e_{k}\right\rangle^{2} \leq|x|^{2}+2 \int_{0}^{t}\left\langle B\left(Y_{s}+Z_{s}\right), Y_{s}\right\rangle d s .
$$

Hence, by assumption (59), for $t \in[0, T]$,

$$
\left|Y_{t}\right|^{2} \leq|x|^{2}+2 C \int_{0}^{t}\left(\left|Y_{s}\right|^{2}+e^{p\left|Z_{s}\right|}+1\right) d s
$$

and therefore, by the Gronwall lemma,

$$
\left|Y_{t}\right|^{2} \leq e^{C T}\left(|x|^{2}+2 C T\left(\sup _{s \in[0, T]} e^{p\left|Z_{s}\right|}+1\right)\right) .
$$


This implies

$$
\mathbb{E}\left[\sup _{t \in[0, T]}\left|Y_{t}\right|^{2}\right] \leq e^{C_{1} T}\left(|x|^{2}+\mathbb{E}\left[\sup _{s \in[0, T]} e^{p\left|Z_{s}\right|}\right]+1\right) .
$$

Therefore,

$$
\begin{aligned}
\mathbb{E}\left[\sup _{t \in[0, T]}\left|X_{t}\right|^{2}\right] & \leq 2 e^{C_{1} T}\left(|x|^{2}+\mathbb{E}\left[\sup _{s \in[0, T]} e^{p\left|Z_{s}\right|}\right]+1\right)+2 \mathbb{E}\left[\sup _{t \in[0, T]}\left|Z_{t}\right|^{2}\right] \\
& \leq e^{C_{p} T}\left(|x|^{2}+\mathbb{E}\left[\sup _{s \in[0, T]} e^{p\left|Z_{s}\right|}\right]+1\right) .
\end{aligned}
$$

\subsection{Proof of Theorem 16}

By Theorem 1 we only have to prove existence of strong solution for $\mu$-a.e. $x \in H$.

We will again consider truncated bounded drifts $B_{N}=B 1_{B(0, N)}, N \geq 1$.

By the main result in [5] there exists a Borel set $\tilde{G} \subset H$ with $\mu(\tilde{G})=1$ such that for any $x \in \tilde{G}$ we have pathwise uniqueness for each stochastic equation

$$
d X_{t}=\left(A X_{t}+B_{N}\left(X_{t}\right)\right) d t+d W_{t}, \quad X_{0}=x \in H,
$$

$N \geq 1$. Let $x \in \tilde{G}$. By the Girsanov theorem (see Appendix in [5]) there exists (a unique in law) weak mild solution $X_{N}=\left(X_{N}(t)\right)=\left(X_{N}(t)\right)_{t \geq 0}$ for each stochastic equation (62).

Therefore we can apply a generalization of the Yamada-Watanabe theorem (see Theorem 2 in [21] and [18]) to (62) when $x \in \tilde{G}$.

Let us fix any filtered probability space $\left(\Omega, \mathcal{F},\left(\mathcal{F}_{t}\right), \mathbb{P}\right)$ on which there is defined a cylindrical $\left(\mathcal{F}_{t}\right)$-Wiener process $W$. By the Yamada-Watanabe theorem, for any $N \geq 1$, on the fixed filtered probability space above there exists a (unique) strong mild solution $X_{N}$ to (62). Moreover, since

$$
B_{N}(x)=B_{N+k}(x), \quad x \in B(0, N),
$$

$k \geq 1$, we have by Theorem 1 that, $\mathbb{P}$-a.s.,

$$
\tau_{N}:=\tau_{N}^{X_{N}}=\tau_{N}^{X_{N+k}}, \quad k \geq 1, N \geq 1,
$$

and $X_{N}\left(t \wedge \tau_{N}\right)=X_{N+k}\left(t \wedge \tau_{N}\right), t \geq 0$.

It is enough to construct the strong solution $X$ to (1) on $[0, T]$ for a fixed $T>0$. We define an $H$-valued stochastic process $X$ on $\Omega^{\prime}=\cup_{N \geq 1}\left\{\tau_{N}>T\right\}$ as

$$
X(t)(\omega):=X_{N}(t)(\omega), \quad t \in[0, T],
$$

if $\omega \in\left\{\tau_{N}>T\right\}$ (we set $X_{t}(\omega)=0$ if $\omega \notin \Omega^{\prime}, t \in[0, T]$ ). Then $X(t)$ is well defined. 
It is not difficult to prove that $X$ is a strong mild solution on $[0, T]$ if we show that

$$
\lim _{N \rightarrow \infty} \mathbb{P}\left(\tau_{N}>T\right)=1
$$

(this will imply that $\mathbb{P}\left(\Omega^{\prime}\right)=1$ ). To verify (63) we will apply Theorem 19, Note that each $B_{N}$ satisfies

$$
\left\langle B_{N}(y+z), y\right\rangle \leq C\left(|y|^{2}+e^{p|z|}+1\right), y, z \in H
$$

with the same constants $C$ and $p$ of (59). By Theorem [19] we obtain

$$
\mathbb{E}\left[\sup _{t \in[0, T]}\left|X_{N}(t)\right|^{2}\right] \leq e^{C_{p} T}\left(|x|^{2}+K_{T}+1\right),
$$

with $C_{p}$ independent of $N \geq 1$. Since

$$
\mathbb{P}\left(\tau_{N} \leq T\right)=\mathbb{P}\left(\sup _{t \in[0, T]}\left|X_{N}(t)\right| \geq N\right)
$$

by (64) and the Chebychev inequality we easily get assertion (63) from (64) and this completes the proof.

\subsection{Existence and uniqueness of local mild solutions}

Finally, let us discuss a possible extension of our result to the case when the drift term $B$ only belongs to $B_{b, l o c}(H, H)$, without requiring hypothesis (59).

We need the concept of local solution (see, for instance, [1] for some additional facts about local solutions). Let $\left(\Omega, \mathcal{F},\left(\mathcal{F}_{t}\right), \mathbb{P}\right)$ be a filtered probability space. A stopping time $\tau: \Omega \rightarrow[0,+\infty]$ is called accessible if there exists a sequence of stopping times $\left(\tau_{n}\right)=\left(\tau_{n}\right)_{n \in \mathbb{N}}$ such that $\mathbb{P}\left(\tau_{n}<\tau\right)=1$ and $\mathbb{P}\left(\lim _{n \rightarrow \infty} \tau_{n}=\tau\right)=1$. The previous sequence $\left(\tau_{n}\right)$ is called an approximating sequence of $\tau$.

Notice that, if $\tau_{1}$ and $\tau_{2}$ are accessible stopping times, then also $\tau=\tau_{1} \wedge \tau_{2}$ is an accessible stopping time.

Let $\tau$ be an accessible stopping time and consider $[0, \tau) \times \Omega=\{(t, \omega) \in[0,+\infty) \times \Omega$ : $0 \leq t<\tau(\omega)\}$. An $H$-valued stochastic process $X$ defined on $[0, \tau)($ i.e., $X:[0, \tau) \times \Omega \rightarrow$ $H$ ) is called $\left(\mathcal{F}_{t}\right)$-adapted if $X_{t}(\cdot):\{t<\tau\} \rightarrow H$ is $\mathcal{F}_{t}$-measurable, for any $t \geq 0$ (on $\{t<\tau\}$ we consider the restricted $\sigma$-algebra $\left.\{A \cap\{t<\tau\}\}_{A \in \mathcal{F}_{t}}\right)$; moreover, it is called continuous if trajectories are continuous on $[0, \tau), \mathbb{P}$-a.s.. Note that $X$ is $\left(\mathcal{F}_{t}\right)$-adapted if and only if the process $\tilde{X}=\left(\tilde{X}_{t}\right)_{t \geq 0}$,

$$
\tilde{X}_{t}=X_{t} 1_{\{\tau>t\}}+0 \cdot 1_{\{\tau \leq t\}}
$$

is $\left(\mathcal{F}_{t}\right)$-adapted. 
Definition 2. Let $x \in H$. We call local weak mild solution to (1) a tuple $\left(\Omega, \mathcal{F},\left(\mathcal{F}_{t}\right)\right.$, $\mathbb{P}, W, X, \tau)$, where $\left(\Omega, \mathcal{F},\left(\mathcal{F}_{t}\right), \mathbb{P}\right)$ is a filtered probability space on which it is defined a cylindrical $\left(\mathcal{F}_{t}\right)$-Wiener process $W$, an accessible stopping time $\tau$ and a continuous $\left(\mathcal{F}_{t}\right)$-adapted $H$-valued process $X$ defined on $[0, \tau)$ such that, there exists an approximating sequence $\left(\tau_{n}\right)$ of $\tau$ for which, $\mathbb{P}$-a.s., on $\left\{t \leq \tau_{n}\right\}$

$$
X_{t}=e^{t A} x+\int_{0}^{t} e^{(t-s) A} B\left(X_{s}\right) d s+\int_{0}^{t} e^{(t-s) A} d W_{s}
$$

for all $n \in \mathbb{N}$ and $t \geq 0$.

A local weak mild solution $X$ which is $\left(\overline{\mathcal{F}}_{t}^{W}\right)$-adapted (here $\left(\overline{\mathcal{F}}_{t}^{W}\right)$ denotes the completed natural filtration of the cylindrical process $W$ ) and such that $\tau$ is an $\left(\overline{\mathcal{F}}_{t}^{W}\right)$ stopping time is called a local strong mild solution.

Theorem 20. Assuming Hypothesis 1 and $B \in B_{b, l o c}(H, H)$, existence of local strong mild solutions holds for $\mu$-a.e. initial condition $x \in H$. Moreover, for $\mu$-a.e. $x \in H$, if $X$ and $Y$ are two local weak mild solutions on the same $\left(\Omega, \mathcal{F},\left(\mathcal{F}_{t}\right), \mathbb{P}\right)$ with the same cylindrical Wiener process $W$, defined on $\left[0, \tau^{X}\right)$ and $\left[0, \tau^{Y}\right)$ respectively $\left(\tau^{X}\right.$ and $\tau^{Y}$ are accessible stopping times as in Definition 2$)$, then, $\mathbb{P}$-a.s., $X=Y$ on $[0, \tau)$, where $\tau=\tau^{X} \wedge \tau^{Y}$

Proof. Let us sketch some of the details of the proof.

From the first part of the proof of Theorem 16 (see also Theorem 1 in [5]), given apriori a filtered probability space $\left(\Omega, \mathcal{F},\left(\mathcal{F}_{t}\right), \mathbb{P}\right)$ and a cylindrical $\left(\mathcal{F}_{t}\right)$-Wiener process $W$, we have the existence of a local strong mild solution $X_{N}$ on $\left[0, \tau_{N}\right)$, for every $N \in \mathbb{N}$. Note that each $\tau_{N}$ is accessible since as approximating sequence we may take $\tau_{N, n}=\inf \left\{t \geq 0: X_{N}(t) \notin B\left(0, N-\frac{1}{n}\right)\right\} \wedge n$, for $n \geq 1$.

Thus, taking $\tau=\sup _{N \in \mathbb{N}} \tau_{N}, \tau$ is accessible and we have a local strong mild solution on $[0, \tau)$.

In order to prove uniqueness, we first note that if $X$ is a local weak mild solution defined on $\left[0, \tau^{X}\right)$ and $\left(\tau_{n}^{X}\right)$ is an approximating sequence of $\tau^{X}$ as in the definition of solution, then assertion (28) of Theorem 10 holds, for any $t>0, n \geq 1, \mathbb{P}$-a.s., on the event $\left\{t \leq \tau_{n, N}^{X}\right\}$, where $\tau_{n, N}^{X}$ is the stopping time

$$
\tau_{n, N}^{X}=\inf \left\{t \in\left[0, \tau^{X}\right): X_{t} \notin B(0, N)\right\} \wedge \tau_{n}^{X}
$$

$\left(\tau_{n, N}^{X}=\tau_{n}^{X}\right.$ if the set is empty; to show that $\tau_{n, N}^{X}$ is a stopping time, note that $\tau_{n, N}^{X}=$ $\inf \left\{t \geq 0: \tilde{X}_{t} \notin B(0, N)\right\} \wedge \tau_{n}^{X}$, where $\tilde{X}_{t}$ is defined in (65) with $\tau$ replaced by $\tau^{X}$ ).

Indeed, one can repeat the arguments in the proof of Theorem 10 with the same functions $u_{N}$, replacing $\tau_{N}^{X}$ with $\tau_{n, N}^{X}$.

Now let $X$ and $Y$ be two local weak mild solutions as in the second part of the theorem. 
If $\left(\tau_{n}^{X}\right)$ and $\left(\tau_{n}^{Y}\right)$ are, respectively, approximating sequences of $\tau^{X}$ and $\tau^{Y}$ as in the definition of solution, then in order to prove uniqueness, it is enough to consider $\sigma_{n}=\tau_{n}^{X} \wedge \tau_{n}^{Y}$ and check that, $\mathbb{P}$-a.s.,

$$
X=Y \text { on }\left[0, \sigma_{n}\right], n \geq 1 .
$$

Let us fix $n \geq 1$. We can adapt the proof of Theorem 1, arguing on the interval $\left[0, \eta \wedge T \wedge \tau_{n, N}^{X} \wedge \tau_{n, N}^{Y}\right]$ (cf. (49) $)$. We finally get that $X=Y$ on $\left[0, T \wedge \sigma_{n}\right], T>0$, and this gives the assertion.

Acknowledgement. The authors would like to thank the referees for their useful remarks and suggestions.

\section{References}

[1] Albeverio, S., Brzezniak, Z., Wu, J.F.: Existence of global solutions and invariant measures for stochastic differential equations driven by Poisson type noise with non-Lipschitz coefficients, J. Math. Anal. Appl. 371, 309-322 (2010)

[2] Chojnowska-Michalik, A., Goldys, B.: Generalized Ornstein-Uhlenbeck Semigroups: Littlewood-Paley-Stein Inequalities and the P. A. Meyer Equivalence of Norms. J. Funct. Anal. 182, 243-279 (2001)

[3] Chojnowska-Michalik, A., Goldys, B.: Symmetric Ornstein-Uhlenbeck Semigroups and their Generator. Probab. Theory Relat. Fields 124, 459-486 (2002)

[4] Da Prato, G.: Kolmogorov equations for stochastic PDEs. Advanced Courses in Mathematics. CRM Barcelona. Birkhäuser Verlag, Basel (2004)

[5] Da Prato, G., Flandoli, F., Priola, E., Röckner, M.: Strong uniqueness for stochastic evolution equations in Hilbert spaces perturbed by a bounded measurable drift, Annals of Probability 41, 3306-3344 (2013). doi: 10.1214/12-AOP763

[6] Da Prato, G., Zabczyk, J.: Stochastic equations in infinite dimensions, Encyclopedia of Mathematics and its Applications, 44. Cambridge University Press, Cambridge (1992)

[7] Da Prato, G., Zabczyk, J.: Second Order Partial Differential Equations in Hilbert Spaces, London Math. Soc. Lecture Note Ser., vol. 293, Cambridge University Press, Cambridge (2002)

[8] Da Prato, G., Flandoli, F., Pathwise uniqueness for a class of SDEs in Hilbert spaces and applications, J. Funct. Anal. 259, 243-267 (2010)

[9] Fedrizzi, E.: thesis, Pisa (2009) 
[10] Fedrizzi, E., Flandoli, F.: Pathwise uniqueness and continuous dependence for SDEs with nonregular drift, Stochastics 83, 241-257 (2011)

[11] Ferrario, B.: Uniqueness and absolute continuity for semilinear SPDEs, to appear in the Proceedings of "Seminar on Stochastic Analysis, Random Fields and Applications-VII", Ascona, Birkhäuser (2011)

[12] Flandoli, F.: Random perturbation of PDEs and fluid dynamic models, Lecture Notes in Mathematics 2015, Springer, Berlin (2011)

[13] Flandoli, F., Gubinelli, M., Priola, E.: Well-posedness of the transport equation by stochastic perturbation, Invent. Math. 180, 1-53 (2010)

[14] Gatarek, D., Goldys, B.: On solving stochastic evolution equations by the change of drift with application to optimal control, "Stochastic partial differential equations and applications" (Trento, 1990), 180-190, Pitman Res. Notes Math. Ser., 268, Longman Sci. Tech., Harlow (1992)

[15] Gyöngy, I., Krylov N. V.: Existence of strong solutions for Itô's stochastic equations via approximations, Probab. Theory Relat. Fields 105, 143-158 (1996)

[16] Gyöngy, I., Martínez, T.: On stochastic differential equations with locally unbounded drift, Czechoslovak Math. J. 51 (126), 763-783 (2001)

[17] Krylov, N. V., Röckner, M.: Strong solutions to stochastic equations with singular time dependent drift, Probab. Theory Relat. Fields 131, 154-196 (2005)

[18] Liu, W., Röckner, M.: Introduction to Stochastic Partial Differential Equations, in preparation.

[19] Ma, Z. M., Röckner, M.: Introduction to the theory of (nonsymmetric) Dirichlet forms, Universitext. Springer-Verlag, Berlin, 1992

[20] Maas, J., van Neerven, J.M.A.M.: Gradient estimates and domain identification for analytic Ornstein-Uhlenbeck operators, Nonlinear Parabolic Problems: Herbert Amann Festschrift Progress in Nonlinear Differential Equations and Their Applications, Vol. 60, pp. 463-477. Birkhäuser (2011)

[21] Ondreját, M.: Uniqueness for stochastic evolution equations in Banach spaces. Dissertationes Math. (Rozprawy Mat.) 426 (2004)

[22] Prévôt, C., Röckner, M.: A concise course on stochastic partial differential equations, Lecture Notes in Mathematics, 1905, Springer, Berlin, 2007

[23] Priola, E.: Pathwise uniqueness for singular SDEs driven by stable processes, Osaka J. Math. 49, 421-447 (2012)

[24] Schmuland, B.: Dirichlet forms with polynomial domain, Math. Japon. 37, 10151024 (1992) 
[25] Tanaka, H., Tsuchiya, M., Watanabe, S.: Perturbation of drift-type for Lévy processes, J. Math. Kyoto Univ. 14, 73-92 (1974)

[26] Veretennikov, A. J.: Strong solutions and explicit formulas for solutions of stochastic integral equations, Mat. Sb. (N.S.) 111(153) (3), 434-452 (1980)

[27] Zhang, X.: Strong solutions of SDES with singular drift and Sobolev diffusion coefficients, Stochastic Process. Appl. 115, 1805-1818 (2005)

[28] Zvonkin, A. K.: A transformation of the phase space of a diffusion process that removes the drif, Mat. Sb. (N.S.) 93 (135), 129-149 (1974) 